\title{
Electroacupuncture for Bladder Function Recovery in Patients Undergoing Spinal Anesthesia
}

\author{
Yinqiu Gao, ${ }^{1}$ Xinyao Zhou, ${ }^{2}$ Xichen Dong, ${ }^{1}$ Qing Jia, ${ }^{1}$ Shen Xie, ${ }^{1}$ and Ran Pang ${ }^{3}$ \\ ${ }^{1}$ Division of Anesthesia, Guang An Men Hospital, China Academy of Chinese Medical Sciences, Beijing 100053, China \\ ${ }^{2}$ Division of Internal Medicine, Guang An Men Hospital, China Academy of Chinese Medical Sciences, Beijing 100053, China \\ ${ }^{3}$ Division of Urology, Guang An Men Hospital, China Academy of Chinese Medical Sciences, No. 5 Bei Xian Ge Street, \\ Xicheng District, Beijing 100053, China
}

Correspondence should be addressed to Ran Pang; pangran2002@sina.com

Received 17 September 2014; Accepted 10 December 2014; Published 24 December 2014

Academic Editor: Christian Lehmann

Copyright (C) 2014 Yinqiu Gao et al. This is an open access article distributed under the Creative Commons Attribution License, which permits unrestricted use, distribution, and reproduction in any medium, provided the original work is properly cited.

Purpose. To determine the efficacy of electroacupuncture on recovering postanesthetic bladder function. Materials and Methods. Sixty-one patients undergoing spinal anaesthesia were recruited and allocated into electroacupuncture or control group randomly. Patients in electroacupuncture group received electroacupuncture therapy whereas ones in control group were not given any intervention. Primary endpoint was incidence of bladder overdistension and postoperative urinary retention. Secondary endpoints included time to spontaneous micturition, voided volume, and adverse events. Results. All patients (31 in electroacupuncture group and 30 in control group) completed the evaluation. During postoperative follow-up, patients in electroacupuncture group presented a significant lower proportion of bladder overdistension than counterparts in control group ( $16.1 \%$ versus $53.3 \%, P<0.01)$. However, no significant difference was found in incidence of postoperative urinary retention between the two groups $(0 \%$ versus $6.7 \%$, $P>0.05)$. Furthermore, a shorter time to spontaneous micturition was found in electroacupuncture group compared to control group (228 min versus $313 \mathrm{~min}, P<0.001$ ), whereas urine volume and adverse events had no significant difference between the two groups. Conclusions. Electroacupuncture reduced the proportion of bladder overdistension and shortened the time to spontaneous micturition in patients undergoing spinal anesthesia. Electroacupuncture may be a therapeutic strategy for postanesthetic bladder dysfunction.

\section{Introduction}

Spinal anesthesia is the simplest and most reliable technique in regional anesthesia. It is widely used for lower abdominal, pelvic, perineal, and lower extremity surgery [1]. Compared to general anesthesia, spinal anesthesia has numerous advantages including rapid onset, lower patient discomfort during the procedure, and decreased need for postoperative analgesia $[2,3]$. However, spinal anesthesia is more likely to result in detrusor dysfunction due to blocking the afferent and efferent limbs of the micturition reflex and may subsequently lead to bladder overdistension (BOD). Once bladder is sufficiently overdistended, detrusor contractility remains impaired, which can develop postoperative urinary retention (PUR) [4]. Although urethral catheterization has been believed as the standard care for PUR, catheter-associated urinary tract infection not only lengthens the hospital stay but also increases the mortality [5]. To prevent the PUR, some therapeutic attempts, such as restrictive fluid regimens [6], ingestion of caffeine [7], and administration of $\alpha$ blockers $[8,9]$, have been used in clinical practice. Unfortunately, these strategies cannot meet all the clinical needs due to their limited applying fields. Studies have shown that fluid restriction cannot lower the incidence of PUR in low-risk surgery patients [10, 11]. Caffeine is not suitable for patients with overactive bladder or urinary incontinence, because it may worsen these symptoms [12,13]. Administration of $\alpha$ blockers may lead to severe hypotension for patients undergoing spinal anesthesia because blood pressure lowered by spinal anesthesia [14] may be further 
reduced by $\alpha$ blockers. Therefore, it is necessary to develop a new therapy to facilitate the recovery of postanesthetic bladder function.

Our study aimed to evaluate the effect of electroacupuncture on recovering bladder function in patients undergoing spinal anesthesia.

\section{Methods}

2.1. Study Population. Patients undergoing spinal anesthesia due to arthroscopic knee surgery were recruited from Guang An Men hospital, China Academy of Chinese Medical Sciences between January 2010 and December 2012. Inclusion criteria for the study were patients with American Society of Anesthesia (ASA) Physical Status I or II and age ranging from 18 to 65 years. Exclusion criteria were patients with emergency surgery, history of bladder outlet obstruction, urinary tract infection, spinal or neurological disorders, and coagulation disorders.

The study protocol was approved by the institutional review board in Guang An Men Hospital and it was designed according to the Declaration of Helsinki, the International Conference on Harmonisation. All study participants provided written informed consent.

2.2. Study Design. Patients who met the protocol eligibility criteria were allocated into electroacupuncture or control group randomly, using a random number generator. Spinal anesthesia for all the patients was performed by the same anesthetist. When the level of sensory block regressed to T10 segment, which was checked by pin-prick test, patients in electroacupuncture group received electroacupuncture therapy, whereas ones in control group were not given any intervention. Postoperative bladder volume was monitored by ultrasonography at the end of operation and every subsequent hour until spontaneous micturition. The BOD was defined if a patient's bladder volume was more than $400 \mathrm{~mL}$ before spontaneous micturition. Once the bladder volume was more than $600 \mathrm{~mL}$, PUR was considered and an indwelling urethral catheterization was performed to prevent persistent bladder dysfunction [4]. Primary endpoint was the incidence of BOD and PUR, which were calculated in each group and compared to assess the effect of electroacupuncture. The time to spontaneous micturition after spinal anesthesia, urine volume, and adverse events, as secondary endpoints, were recorded and analyzed. Additionally, the extra analgesia which patients administered and the amount of intravenous fluids before spontaneous micturition in each group were also recorded.

2.3. Spinal Anesthesia. Patient was positioned with lateral decubitus and spinal anesthesia was performed at L3/L4 interspace with a Quincke 22-gauge needle using hyperbaric tetracaine $(0.33 \%) 10 \mathrm{mg}$. Hyperbaric tetracaine was composed of tetracaine $1 \mathrm{~mL}(10 \mathrm{mg})$, ephedrine $1 \mathrm{~mL}$ (30 mg), and $10 \%$ glucose injection $(1 \mathrm{~mL})$. The tetracaine injection speed was $1 \mathrm{~mL}$ per 5 seconds. The maximum level of sensory block was obtained between T6 and T8 segments.
2.4. Electroacupuncture Intervention. The electroacupuncture therapy was performed by the same acupuncturist in operation room. Sterile disposable, stainless filiform acupuncture needles, $0.3 \mathrm{~mm}$ in diameter and $50 \mathrm{~mm}$ in length (HWATO, Suzhou Medical Appliance Factory, Suzhou, China), were used. The selected acupoints included CV3 (Zhong Ji), CV4 (Guan Yuan), and bilateral ST29 (Gui Lai). After the acupuncture needles were inserted into acupoints, Han's acupoint nerve stimulator (model HANS 200E, Beijing Huayun Ante technology Co. Ltd., China) was connected to the handles of acupuncture needles to provide the electrical stimulation for 30 minutes. A low-frequency $(2 \mathrm{~Hz})$ continuous wave stimulation was applied.

2.5. Data Analyses. Statistical analyses were performed using the SPSS software package for Windows version 19.0 (SPSS Inc., IL, USA). Continuous variables were expressed as means \pm standard deviation, while categorical variables were expressed as percentage and frequency. The occurrence rates of BOD and PUR were compared by Pearson's Chi-square test between two groups. Shapiro-Wilk $W$ test was used to detect if the data fitted normal distribution in each group. The time to spontaneous micturition, urine volume, and amount of intravenous fluids between the two groups were compared by two-sample $t$-test if the data followed normal distribution; otherwise, Mann-Whitney $U$ test was used. The frequency of adverse events was analyzed between the two groups using Fisher exact test. All reported $P$ values were two-sided, and $P<0.05$ was considered statistically significant.

\section{Results}

A total of 61 patients ( 31 in electroacupuncture group and 30 in control group) were recruited in our study. Table 1 shows the patients' demographic and baseline (sensory level receded to T10 segment) measurements. No significant difference was found between the two groups $(P>0.05)$. During the procedure, patients' pain was controlled well and no extra analgesia was administered in either group.

During postoperative follow-up, electroacupuncture group showed a significant lower incidence of BOD compared with control group (16.1\% versus $53.3 \%, P<0.01)$. Of patients with BOD, two patients (36-year-old male and 50 -year-old female) in control group developed PUR and were treated with indwelling urethral catheterization, while no patients experienced PUR in electroacupuncture group. There was no significant difference in incidence of PUR between the two groups ( $0 \%$ versus $6.7 \%, P>0.05$ ). Furthermore, patients in electroacupuncture group presented a shorter time to spontaneous micturition compared to counterparts in control group $(P<0.001)$, whereas no significant difference was found in the urine volume and infusion volume between the two groups (Table 2). Additionally, two and six patients complained of the feeling of incomplete emptying until postoperative day one in electroacupuncture and control group, respectively. The frequency of this adverse event did not show significant difference between the two groups (6.5\% versus $21.4 \%, P>0.05)$. 
TABLE 1: Patient characteristics and measurements at baseline.

\begin{tabular}{lcc}
\hline & EA group & Control group \\
\hline Number & 31 & 30 \\
Age (years) & $55 \pm 6$ & $53 \pm 11$ \\
Gender & & \\
$\quad$ Male & 10 & 9 \\
$\quad$ Female & 21 & 21 \\
Height $(\mathrm{cm})$ & $164 \pm 8$ & $166 \pm 8$ \\
Weight $(\mathrm{kg})$ & $69 \pm 11$ & $68 \pm 9$ \\
SBP $(\mathrm{mmHg})$ & $115 \pm 12$ & $114 \pm 13$ \\
DBP (mmHg) & $66 \pm 16$ & $68 \pm 11$ \\
MAP (mmHg) & $83 \pm 14$ & $83 \pm 10$ \\
HR & $72 \pm 8$ & $72 \pm 9$ \\
Duration of surgery (min) & $83.4 \pm 17$ & $85.5 \pm 16.4$ \\
\hline
\end{tabular}

Values are given as mean \pm standard deviation. EA: electroacupuncture; SBP: systolic blood pressure; DBP: diastolic blood pressure; MAP: mean arterial pressure; HR: heart rate.

TABLe 2: Time to spontaneous micturition, urine volume, and infusion volume in the two groups.

\begin{tabular}{lcc}
\hline & EA group & Control group \\
\hline$N$ & 31 & 28 \\
Time to SM $(\mathrm{min})^{\mathrm{a}}$ & $228 \pm 78$ & $313 \pm 91^{*}$ \\
Urine volume $(\mathrm{mL})^{\mathrm{b}}$ & $339 \pm 109$ & $361 \pm 179$ \\
Infusion volume $(\mathrm{mL})^{\mathrm{b}}$ & $1631 \pm 270$ & $1673 \pm 303$ \\
\hline
\end{tabular}

Values are given as mean \pm standard deviation. ${ }^{\mathrm{a}}$ Difference was analyzed by two-sample $t$-test between the two groups; ${ }^{b}$ Difference was analyzed by Mann-Whitney $U$ test between the two groups. ${ }^{*} P<0.001$ versus EA group. EA: electroacupuncture; SM: spontaneous micturition.

In terms of different age group, the younger patients $(\leq 50$ years) had a shorter time to spontaneous micturition than the older ones ( $>50$ years) in electroacupuncture group, while no significant difference was found in control group (Table 3). Moreover, younger and older age group presented similar percentage in patients with $\mathrm{BOD}$ in electroacupuncture group (12.5\% versus $17.4 \%$ ) and control group (50\% versus $55 \%)$, respectively. In addition, no significant difference was found between males and females in either group (Table 4).

\section{Discussion}

The main finding of this study is that electroacupuncture can reduce the occurrence rate of $\mathrm{BOD}$ and shorten the time to spontaneous micturition in patients undergoing spinal anesthesia.

Disturbance of bladder function is a main issue for patients undergoing spinal anesthesia, which may develop BOD and PUR. The definition of BOD and PUR varies in different studies. Some studies focused on ultrasound assessment $[15,16]$, while others relied on the clinical examination $[17,18]$. In order to ensure accuracy of diagnosis, our study used ultrasound to assess bladder volume. Studies have shown that ultrasound can provide an accuracy of $94 \%$ for a predicted bladder volume which is more than
$100 \mathrm{~mL}$ [19] and only has a measurement error of less than $15 \mathrm{~mL}$ [20]. On the other hand, the normal bladder volume remains controversial, ranging from 300 to $600 \mathrm{~mL}$. Although a study showed that bladder volume between 400 and $600 \mathrm{~mL}$ is normal [15], 13\% patients with bladder volume of more than $400 \mathrm{~mL}$ required catheterization after undergoing spinal anesthesia [21]. Urodynamic study revealed that individuals reported bladder distension when their bladder volume reached $400 \mathrm{~mL}$ [22]. Moreover, catheterization is recommended for postoperative patients whose bladder volume is more than $600 \mathrm{~mL}$ [4]. After taking account of the urodynamic finding and the expert recommendation, we used $400 \mathrm{~mL}$ and $600 \mathrm{~mL}$ as bladder volume threshold for bladder BOD and PUR, respectively.

The incidence of PUR in general surgical population is about 4\% [23] and varies according to the type of surgery. In our study, the occurrence rate of PUR in control group was $6.7 \%$ which is lower than the reported $21.1 \%$ to $55 \%[24,25]$ in knee surgery. The main reason probably comes from the different patient characteristics and definition of PUR. Our study excluded patients with bladder outlet obstruction, but those studies did not. As is reported, preexisting bladder outlet obstruction is important risk factor for PUR [26]. The various diagnostic criteria of PUR also contribute to the difference in outcome between our study and others.

A study showed that the level of sensory block took seven to eight hours to regress to S3 segment, which allowed patient to urinate spontaneously, after spinal injection of hyperbaric bupivacaine or tetracaine [27]. Our study showed a less time to spontaneous micturition, which was approximately five hours in control group. The difference in recovery time of micturition mainly resulted from the different dose of local anesthetic in spinal anesthesia. In our study, a moderate dose of tetracaine $(10 \mathrm{mg})$ was adopted, while a high dose of tetracaine $(15 \mathrm{mg}$ ) was used in that study. Another important reason is that a different time point (level of sensory block regressed to T10 segment) was used as baseline to measure the time to spontaneous micturition in our study. Because the level of sensory block varied in different individuals, our study used the time point to minimize measurement bias.

Some studies showed that age and gender were the essential factors for recovery of postanesthetic bladder function. Patients over 50 years and male are more likely to experience BOD and develop PUR after undergoing spinal anesthesia $[16,23,28]$. In our study, the time to spontaneous micturition, urine volume, and the proportion of BOD did not vary by gender or age in control group. The possible reason is that our study ruled out patients with bladder outlet obstruction which always occur in aging or male population. Interestingly, we found that younger patients got faster recovery in bladder function than older ones after they received electroacupuncture therapy. The reason might be that age-related progressive neuronal degeneration delayed the bladder function recovery, which reduced the nerve sensitivity to electroacupuncture therapy.

Acupuncture, as a component of traditional Chinese medicine, has been used for various urological diseases and has gained acceptance among urologists [29]. Some studies have shown its effectiveness on benign prostatic 
TABLE 3: Time to spontaneous micturition, urine volume, and infusion volume in different age group.

\begin{tabular}{|c|c|c|c|c|}
\hline & \multicolumn{2}{|c|}{ EA group } & \multicolumn{2}{|c|}{ Control group } \\
\hline & $\begin{array}{c}\text { Younger age group } \\
(\leq 50 \text { years })\end{array}$ & $\begin{array}{l}\text { Older age group } \\
(>50 \text { years })\end{array}$ & $\begin{array}{c}\text { Younger age group } \\
(\leq 50 \text { years })\end{array}$ & $\begin{array}{c}\text { Older age group } \\
(>50 \text { years }) \\
\end{array}$ \\
\hline$N$ & 8 & 23 & 8 & 20 \\
\hline Time to SM (min) $)^{\mathrm{a}}$ & $167 \pm 40$ & $248 \pm 77^{*}$ & $335 \pm 120$ & $297 \pm 79$ \\
\hline Urine volume $(\mathrm{mL})^{\mathrm{b}}$ & $299 \pm 117$ & $353 \pm 104$ & $276 \pm 139$ & $345 \pm 115$ \\
\hline Infusion volume $(\mathrm{mL})^{\mathrm{b}}$ & $1613 \pm 158$ & $1637 \pm 302$ & $1731 \pm 281$ & $1612 \pm 263$ \\
\hline
\end{tabular}

Values are given as mean \pm standard deviation. ${ }^{\mathrm{a}}$ Difference was analyzed by two-sample $t$-test between the two groups; ${ }^{\mathrm{b}}$ Difference was analyzed by MannWhitney $U$ test between the two groups. ${ }^{*} P<0.001$ versus younger age group. EA: electroacupuncture; SM: spontaneous micturition.

TABLE 4: Time to spontaneous micturition, urine volume, and infusion volume in different gender group.

\begin{tabular}{lcccc}
\hline & & EA group & & \multicolumn{2}{c}{ Control group } \\
& Female & Male & Female & 8 \\
\hline$N$ & 21 & 10 & 20 & $298 \pm 92$ \\
Time to SM (min) & $216 \pm 85$ & $251 \pm 58$ & $314 \pm 122$ & $335 \pm 91$ \\
Urine volume $(\mathrm{mL})^{\mathrm{b}}$ & $333 \pm 111$ & $351 \pm 106$ & $1578 \pm 220$ & $355 \pm 133$ \\
Infusion volume $(\mathrm{mL})^{\mathrm{b}}$ & $1667 \pm 309$ & $1555 \pm 144$ & $1818 \pm 316$ \\
\hline
\end{tabular}

Values are given as mean \pm standard deviation. ${ }^{\mathrm{a}}$ Difference was analyzed by two-sample $t$-test between the two groups; ${ }^{\mathrm{b}}$ Difference was analyzed by MannWhitney $U$ test between the two groups. EA: electroacupuncture; SM: spontaneous micturition.

hyperplasia [30], nocturnal enuresis [31], premature ejaculation [32], and urinary incontinence $[33,34]$. In our study, we found that electroacupuncture could reduce occurrence rate of BOD and shorten the time to spontaneous micturition. The possible mechanism is the modulation of acupuncture on detrusor contraction. An experimental study demonstrated that needling CV4 (Guan Yuan) could increase the frequency of detrusor contraction in rat model of urinary retention [35] and another study showed that needling CV3 (Zhong Ji) could regulate the vesical pressure in rabbit [36]. It was shown in a clinical study that needling ST29 (Gui Lai) could increase the average urine flow and reduce the postvoid residual urine volume in patients undergoing radical hysterectomy [37]. On the other hand, analgesic effect of acupuncture may also contribute to patients' spontaneous micturition which is disturbed by postoperative pain [38]. Some studies have confirmed the role of acupuncture in postoperative pain management [39-41]. The potential mechanism includes inhibition on conduction of pain signals, release of endorphin, activation of analgesic system, and central modulation [42].

To confirm therapeutic effect of electroacupuncture, blank control was used in our study. Although placebo control serves as the golden standard in clinical trials, no standard approach for acupuncture placebo has been published. Sham acupuncture is a control strategy which has been used in clinical trials. However, a systematic review implies that sham acupuncture may have equal therapeutic effect to real acupuncture [43]. Furthermore, patients with experience of acupuncture treatment can distinguish between real and sham acupuncture [44], which may introduce bias to acupuncture study. Therefore, sham acupuncture was not used as control in our study.
The important limitation of this study includes small sample size and nonblind design. Another limitation is lack of the urodynamic evaluation for bladder function. However, it would cause patients' discomfort and is also a burden for both patients and the health insurance.

\section{Conclusions}

Findings from this study suggest that electroacupuncture reduced the incidence of $\mathrm{BOD}$ and shortened the time to spontaneous micturition in patients undergoing spinal anesthesia. Electroacupuncture might be a therapeutic strategy for bladder dysfunction secondary to spinal anesthesia.

\section{Conflict of Interests}

The authors clarify that there is no conflict of interests with any financial organization regarding the material discussed in the paper.

\section{Authors' Contribution}

Yinqiu Gao and Xinyao Zhou contributed equally to this paper.

\section{Acknowledgments}

This work was supported by Grants 2009S189 and ZZ070839, China Academy of Chinese Medical Sciences. The authors are grateful to all the patients who participated in this study. 


\section{References}

[1] M. F. Mulroy and F. V. Salinas, "Neuraxial techniques for ambulatory anesthesia," International Anesthesiology Clinics, vol. 43, no. 3, pp. 129-141, 2005.

[2] S. S. Liu, W. M. Strodtbeck, J. M. Richman, and C. L. Wu, "A comparison of regional versus general anesthesia for ambulatory anesthesia: a meta-analysis of randomized controlled trials," Anesthesia and Analgesia, vol. 101, no. 6, pp. 1634-1642, 2005.

[3] A. Rastogi, P. Gyanesh, S. Nisha, A. Agarwal, P. Mishra, and A. K. Tiwari, "Comparison of general anaesthesia versus regional anaesthesia with sedation in selected maxillofacial surgery: a randomized controlled trial," Journal of Cranio-Maxillo-Facial Surgery, vol. 42, no. 3, pp. 250-254, 2014.

[4] G. Baldini, H. Bagry, A. Aprikian, and F. Carli, "Postoperative urinary retention: anesthetic and perioperative considerations," Anesthesiology, vol. 110, no. 5, pp. 1139-1157, 2009.

[5] C. Chant, O. M. Smith, J. C. Marshall, and J. O. Friedrich, "Relationship of catheter-associated urinary tract infection to mortality and length of stay in critically ill patients: a systematic review and meta-analysis of observational studies," Critical Care Medicine, vol. 39, no. 5, pp. 1167-1173, 2011.

[6] T. Toyonaga, M. Matsushima, N. Sogawa et al., "Postoperative urinary retention after surgery for benign anorectal disease: potential risk factors and strategy for prevention," International Journal of Colorectal Disease, vol. 21, no. 7, pp. 676-682, 2006.

[7] D. Leach, J. Spaulding, J. Thomas, C. Conn, and M. Kutash, “The effect of caffeine on postoperative urinary retention after joint replacement surgery," Orthopaedic Nursing, vol. 32, no. 5, pp. 282-285, 2013.

[8] M. Mohammadi-Fallah, S. Hamedanchi, and A. Tayyebi-Azar, "Preventive effect of tamsulosin on postoperative urinary retention," Korean Journal of Urology, vol. 53, no. 6, pp. 419-423, 2012.

[9] N. N. Gönüllü, M. Dülger, N. Z. Utkan, N. Z. Cantürk, and A. Alponat, "Prevention of postherniorrhaphy urinary retention with prazosin," The American Surgeon, vol. 65, no. 1, pp. 55-58, 1999.

[10] D. J. Pavlin, E. G. Pavlin, D. R. Fitzgibbon, M. E. Koerschgen, and T. M. Plitt, "Management of bladder function after outpatient surgery," Anesthesiology, vol. 91, no. 1, pp. 42-50, 1999.

[11] T. Feliciano, J. Montero, M. McCarthy, and M. Priester, "A retrospective, descriptive, exploratory study evaluating incidence of postoperative urinary retention after spinal anesthesia and its effect on PACU discharge," Journal of Perianesthesia Nursing, vol. 23, no. 6, pp. 394-400, 2008.

[12] S. Lohsiriwat, M. Hirunsai, and B. Chaiyaprasithi, "Effect of caffeine on bladder function in patients with overactive bladder symptoms," Urology Annals, vol. 3, no. 1, pp. 14-18, 2011.

[13] N. J. Davis, C. P. Vaughan, T. M. Johnson II et al., "Caffeine intake and its association with urinary incontinence in United States men: results from national health and nutrition examination surveys 2005-2006 and 2007-2008," Journal of Urology, vol. 189, no. 6, pp. 2170-2174, 2013.

[14] B. Hartmann, A. Junger, J. Klasen et al., "The incidence and risk factors for hypotension after spinal anesthesia induction: an analysis with automated data collection," Anesthesia and Analgesia, vol. 94, no. 6, pp. 1521-1529, 2002.

[15] L. Lamonerie, E. Marret, A. Deleuze, N. Lembert, M. Dupont, and F. Bonnet, "Prevalence of postoperative bladder distension and urinary retention detected by ultrasound measurement," British Journal of Anaesthesia, vol. 92, no. 4, pp. 544-546, 2004.
[16] H. Keita, E. Diouf, F. Tubach et al., "Predictive factors of early postoperative urinary retention in the postanesthesia care unit," Anesthesia and Analgesia, vol. 101, no. 2, pp. 592-596, 2005.

[17] D. Kemp and N. Tabaka, "Postoperative urinary retention: part I-overview and implications for the postanesthesia care unit nurse," Journal of Post Anesthesia Nursing, vol. 5, no. 5, pp. 338341, 1990.

[18] J. G. Petros, E. B. Rimm, R. J. Robillard, and O. Argy, "Factors influencing postoperative urinary retention in patients undergoing elective inguinal herniorrhaphy," American Journal of Surgery, vol. 161, no. 4, pp. 431-433, 1991.

[19] L. S. Marks, F. J. Dorey, M. L. Macairan, C. Park, and J. B. deKernion, "Three-dimensional ultrasound device for rapid determination of bladder volume," Urology, vol. 50, no. 3, pp. 341-348, 1997.

[20] D. J. Pavlin, S. E. Rapp, N. L. Polissar, J. A. Malmgren, M. Koerschgen, and H. Keyes, "Factors affecting discharge time in adult outpatients," Anesthesia and Analgesia, vol. 87, no. 4, pp. 816-826, 1998.

[21] M. F. Mulroy, F. V. Salinas, K. L. Larkin, and N. L. Polissar, "Ambulatory surgery patients may be discharged before voiding after short-acting spinal and epidural anesthesia," Anesthesiology, vol. 97, no. 2, pp. 315-319, 2002.

[22] J. J. Wyndaele, "The normal pattern of perception of bladder filling during cystometry studied in 38 young healthy volunteers," The Journal of Urology, vol. 160, no. 2, pp. 479-481, 1998.

[23] T. Tammela, M. Kontturi, and O. Lukkarinen, "Postoperative urinary retention. I. Incidence and predisposing factors," Scandinavian Journal of Urology and Nephrology, vol. 20, no. 3, pp. 197-201, 1986.

[24] P. Kumar, K. Mannan, A. M. Chowdhury, K. C. Kong, and J. Pati, "Urinary retention and the role of indwelling catheterization following total knee arthroplasty," International Brazilian Journal of Urology, vol. 32, pp. 31-34, 2006.

[25] S. M. Sarasin, M. J. Walton, H. P. Singh, and D. I. Clark, "Can a urinary tract symptom score predict the development of postoperative urinary retention in patients undergoing lower limb arthroplasty under spinal anaesthesia? A prospective study," Annals of the Royal College of Surgeons of England, vol. 88, no. 4, pp. 394-398, 2006.

[26] D. M. Darrah, T. L. Griebling, and J. H. Silverstein, "Postoperative urinary retention," Anesthesiology Clinics, vol. 27, no. 3, pp. 465-484, 2009.

[27] K. Axelsson, K. Mollefors, J. O. Olsson, G. Lingårdh, and B. Widman, "Bladder function in spinal anaesthesia," Acta Anaesthesiologica Scandinavica, vol. 29, no. 3, pp. 315-321, 1985.

[28] H. Lau and B. Lam, "Management of postoperative urinary retention: a randomized trial of in-out versus overnight catheterization," ANZ Journal of Surgery, vol. 74, no. 8, pp. 658661, 2004.

[29] H. Tempest, J. Reynard, R. J. Bryant, F. C. Hamdy, and S. Larré, "Acupuncture in urological practice-a survey of urologists in England," Complementary Therapies in Medicine, vol. 19, no. 1, pp. 27-31, 2011.

[30] Y. Wang, B. Liu, J. Yu, J. Wu, J. Wang, and Z. Liu, "Electroacupuncture for moderate and severe benign prostatic hyperplasia: a randomized controlled trial," PLoS ONE, vol. 8, no. 4, Article ID e59449, 2013.

[31] E. E. Moursy, N. F. Kamel, and A. F. Kaseem, "Combined laser acupuncture and desmopressin for treating resistant cases of 
monosymptomatic nocturnal enuresis: a randomized comparative study," Scandinavian Journal of Urology, vol. 48, no. 6, pp. 559-564, 2014.

[32] D. Sunay, M. Sunay, Y. Aydoğmuş et al., "Acupuncture versus paroxetine for the treatment of premature ejaculation: a randomized, placebo-controlled clinical trial," European Urology, vol. 59, no. 5, pp. 765-771, 2011.

[33] J. H. Kim, D. Nam, M. K. Park, E. S. Lee, and S. H. Kim, "Randomized control trial of hand acupuncture for female stress urinary incontinence," Acupuncture and Electro-Therapeutics Research, vol. 33, no. 3-4, pp. 179-192, 2008.

[34] C. Jin, X. Zhou, and R. Pang, "Effect of electroacupuncture combined with Tolterodine on treating female mixed Urinary Incontinence," Journal of Wound, Ostomy and Continence Nursing, vol. 41, no. 3, pp. 268-272, 2014.

[35] J. H. Wang, B. G. Chen, J. Yin, X. J. Luo, W. G. Zou, and X. M. Zhang, "Influences of electroacupuncture at Guanyuan (CV4) acupoint on the excitability of detrusor of rats with urinary retention after spinal cord injury," Journal of Hubei University of Chinese Medicine, vol. 11, p. 3, 2009.

[36] X. Meng, J. Han, X. Ye, and X. Huang, "Effect of acupuncture on rabbit bladder with urodynamic indexes," Journal of Traditional Chinese Medicine, vol. 33, no. 1, pp. 103-109, 2013.

[37] W. M. Yi, J. J. Li, X. M. Lu, L. L. Jin, A. Z. Pan, and Y. Q. Zou, "Effects of electroacupuncture on urinary bladder function after radical hysterectomy," Chinese Acupuncture \& Moxibustion, vol. 28, no. 9, pp. 653-655, 2008.

[38] R. G. Rogers and L. L. Leeman, "Postpartum genitourinary changes," The Urologic Clinics of North America, vol. 34, no. 1, pp. 13-21, 2007.

[39] Y. Sun, T. J. Gan, J. W. Dubose, and A. S. Habib, "Acupuncture and related techniques for postoperative pain: a systematic review of randomized controlled trials," British Journal of Anaesthesia, vol. 101, no. 2, pp. 151-160, 2008.

[40] W. Meissner, "The role of acupuncture and transcutaneouselectrical nerve stimulation for postoperative pain control," Current Opinion in Anaesthesiology, vol. 22, no. 5, pp. 623-626, 2009.

[41] S. Gavronsky, R. Koeniger-Donohue, J. Steller, and J. W. Hawkins, "Postoperative pain: acupuncture versus percutaneous electrical nerve stimulation," Pain Management Nursing, vol. 13, no. 3, pp. 150-156, 2012.

[42] E. Ernst, "Acupuncture-a critical analysis," Journal of Internal Medicine, vol. 259, no. 2, pp. 125-137, 2006.

[43] H. H. Moffet, "Sham acupuncture may be as efficacious as true acupuncture: a systematic review of clinical trials," The Journal of Alternative and Complementary Medicine, vol. 15, no. 3, pp. 213-216, 2009.

[44] C. R. Dilli, R. Childs, J. Berk et al., "Does prior acupuncture exposure affect perception of blinded real or sham acupuncture?" Acupuncture in Medicine, vol. 32, pp. 155-159, 2014. 


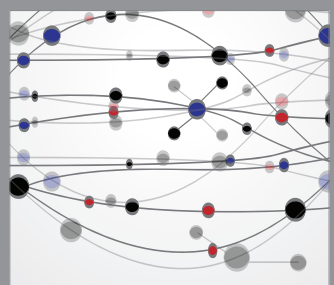

The Scientific World Journal
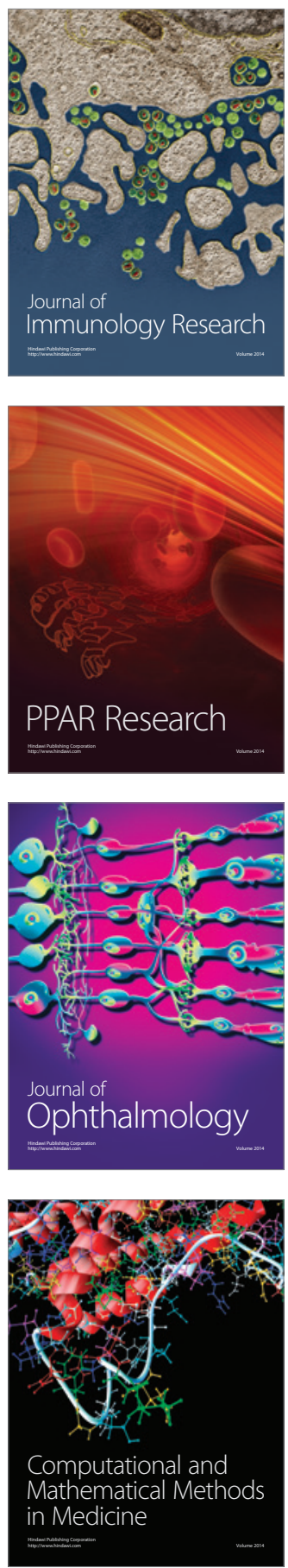

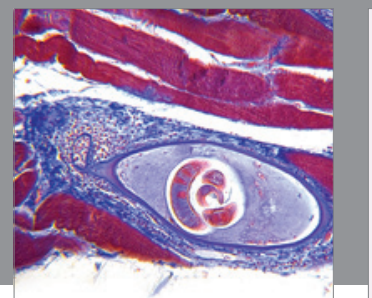

Gastroenterology

Research and Practice
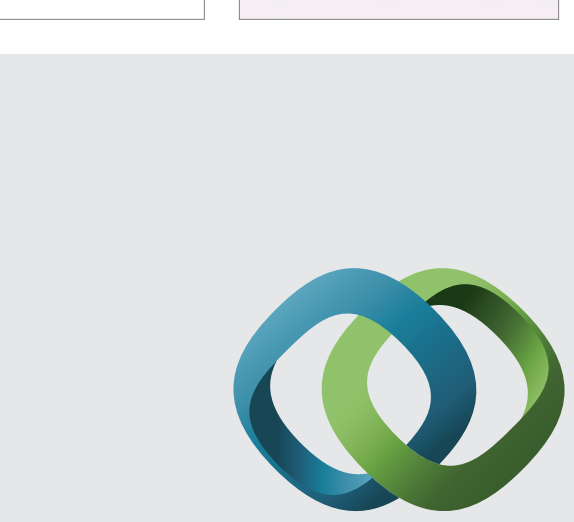

\section{Hindawi}

Submit your manuscripts at

http://www.hindawi.com
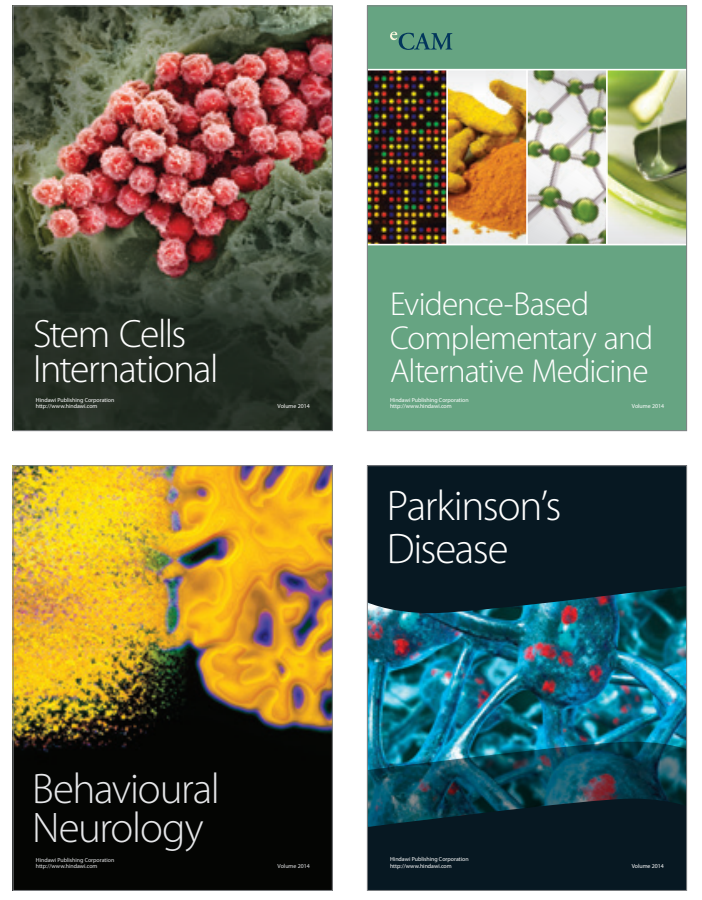
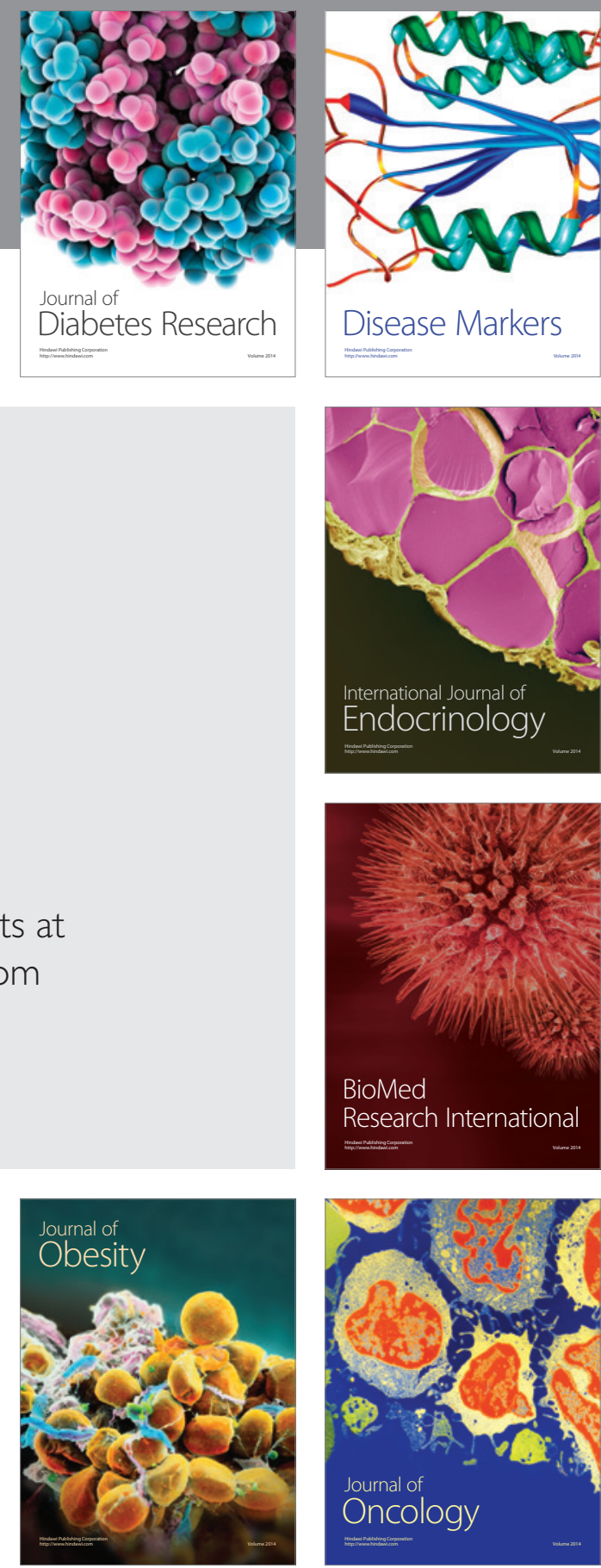

Disease Markers
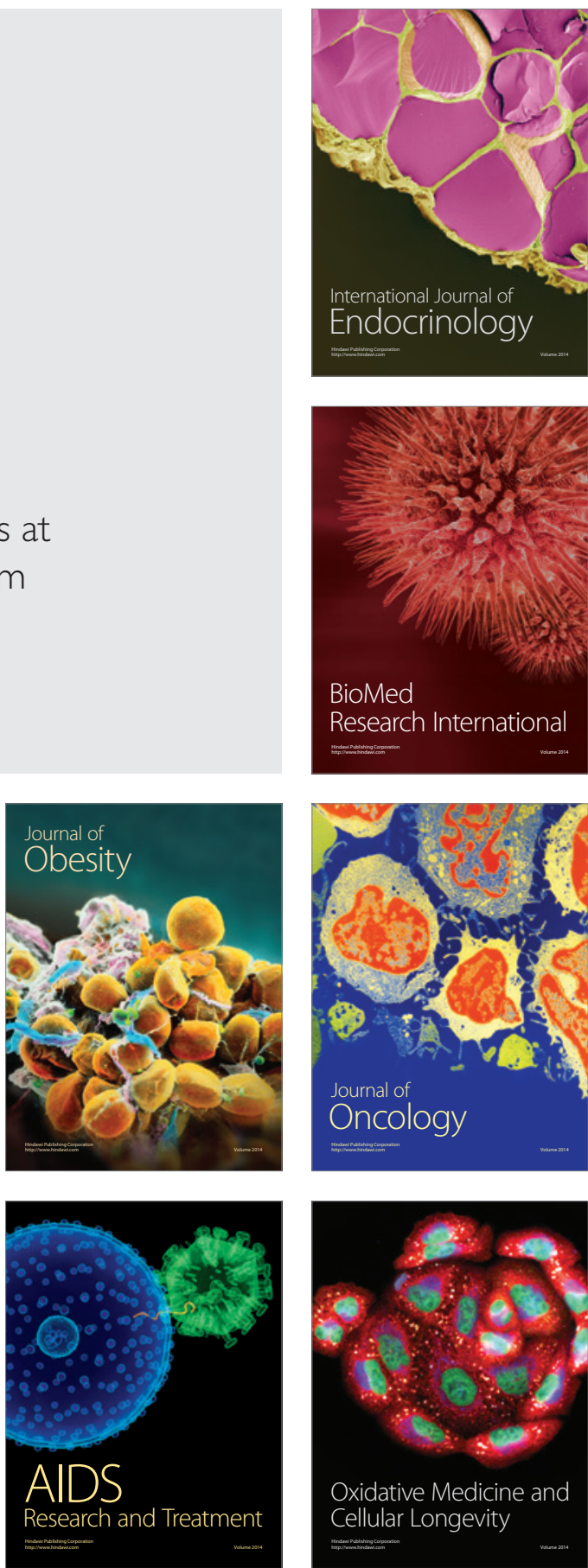